International Mathematical Forum, Vol. 9, 2014, no. 23, 1109 - 1130

HIKARI Ltd, www.m-hikari.com

http://dx.doi.org/10.12988/imf.2014.45103

\title{
Artisan Procedures to Generate Uniform Tilings
}

\author{
Miguel Carlos Fernández-Cabo \\ Departamento de Tecnología y Construcciones Arquitectónicas \\ ETS de Arquitectura, Universidad Politécnica de Madrid \\ C/ GeneralYagüe, 30, $2^{\circ}$ B, 28020 Madrid, Spain
}

Copyright (C) 2014 Miguel Carlos Fernández-Cabo. This is an open access article distributed under the Creative Commons Attribution License, which permits unrestricted use, distribution, and reproduction in any medium, provided the original work is properly cited.

\begin{abstract}
This paper try to prove how artisans could discover all uniform tilings and very interesting others using artisanal combinatorial procedures without having to use mathematical procedures out of their reach. Plane Geometry started up his way through History by means of fundamental drawing tools: ruler and compass. Artisans used same tools to carry out their ornamental patterns but at some point they began to work manually using physical representations of figures or tiles previously drawing by means of ruler and compass. That is an important step for craftsman because this way provides tools that let him come in the world of symmetry operations and empirical knowledge of symmetry groups. Artisans started up to produce little wooden, ceramic or clay tiles and began to experiment with them by means of joining pieces whether edge to edge or vertex to vertex in that way so it can cover the plane without gaps. Economy in making floor or ceramic tiles could be most important reason to develop these procedures. This empiric way to develop tilings led not only to discover all uniform tilings but later discovering of aperiodic tilings.
\end{abstract}

Keywords: Tessellations, tilings, ornament, regular polygons, ornamental patterns, ornamental geometry

\section{INTRODUCTION}

This paper does not try to be a strict mathematical analysis into the tilings's field but to prove artisans could discover through History all uniform tilings with only simple artisanal procedures. Not only the 3 regular tilings but the 8 semi-regular tilings, also 
known like Archimedean tessellations, in addition to others tilings that were found in the way before mathematicians had building a specific scientist body. Most interesting issue in this paper consists of handing methods might lead to discovering various and different tessellations, whether satisfying requirements of utmost regularity or not. Using tessellations like work tools to lay out ornamental or architectonical patterns are the aim for artisans.

We will use lexicon and nomenclature from Krötenheerdt (1969) and Grünbaum and Shephard (1987) for developing of this paper. Grünbaum and Shephard's nomenclature continue the previous works of Cundy y Rollett (1951) and is most extended and agreed in scientist literature even though afterward have appeared others ones trying to simplify and improve nomenclature (proposal of EGICAD team from Cantabria University).

To get an idea of how it might generate all regular and semi-regular tessellations by means of artisanal procedures here we will show the following method inspired in artisans working habits. Experimental basis consists of searching for regular convex polygon combinations by means of joining $n$-gons either edge-to-edge or vertex-tovertex making simples symmetry operations like reflection. We refer primary to any $n-$ gon when is regular convex polygon and is capable to generate tessellations. To start with this method we must begin drawing a circle and dividing it in $n$ equal parts to get a regular $n$-gon and then make material tiles to experiment with them. We will start with the minor edges $n$-gon and then we will progress getting $n$-gons with a more number of edges. We will begin dividing a circle in 3 equal parts for $n$-gons of 1 or 2 edges does not exists. For that will make marks in the circle without move the compass gap. Connecting these points of intersection we will get a regular hexagon and connecting these intersections alternately we will get a regular triangle. Afterward we will show how to get any type of regular polygons and the problems to get any of them. Now we will start with the regular triangle.

\section{TILINGS STARTING FROM PRMITIVES}

\subsection{Triangle-primary}

We will start joining 2 triangles edge-to-edge (fig. 1a). This simple operation allows us to get a group of 2 triangles forming a diamond with all 4 equal edges. Diamond is a cuasi-regular $n$-gon, iso-edge but not iso-angle, though its angles are equals 2 by 2 . If we take this diamond like prototile we verify that this tile cover the plane by means of simple translations. This tessellation will be similar to a regular square tiling which main directions forms other angles than $\pi / 2$ (fig. 1b). Arranging diamonds in a row we get a linear sequence and if this linear sequence can be pack in columns without gaps will get a way to configure a sample of translational tiling in the plane. Indeed, In case we only are searching for covering the plane it is not necessary the prototile be regular polygon. If we proceed to draw tangent circles inscribed into diamonds will get a circle 
packing though these circles will be not tangents each other. To get these circles be tangents it would be necessary move columns or rows. The problem is if we get tangency in rows we cannot get tangency in columns and vice versa. However, in terms of tiling the plane, rows or columns displacements in such tessellations go on covering the plane. Another way to find utter tangency of inscribed circles in diamonds would be modifying main directions angles to get exactly $\pi / 2$, which would be equal to regular square tiling. This idea leads to think about features where exist tilings which can modify vertices angles range keeping tessellation in the plane by means of simple transformations. To a tessellation can allow this type of transformations it is necessary that alignment of tiles edges follow straight lines to infinity, which is but to say that tilings can be building be means of straight lines sets configuring a parallel lines pattern directed in the same direction. Only few tilings fulfill this feature: regular tiling of triangles $\left(3^{6}\right)$, uniform tiling of hexagons and triangles (3.6.3.6), regular square tiling $\left(4^{4}\right)$, cuasi-regular diamond tiling and sliding tilings (sliding strips).

If we follow attaching triangles around the first primary triangle we will get a set of 4 triangles which also configure a regular triangle. If we consider this figure like a new

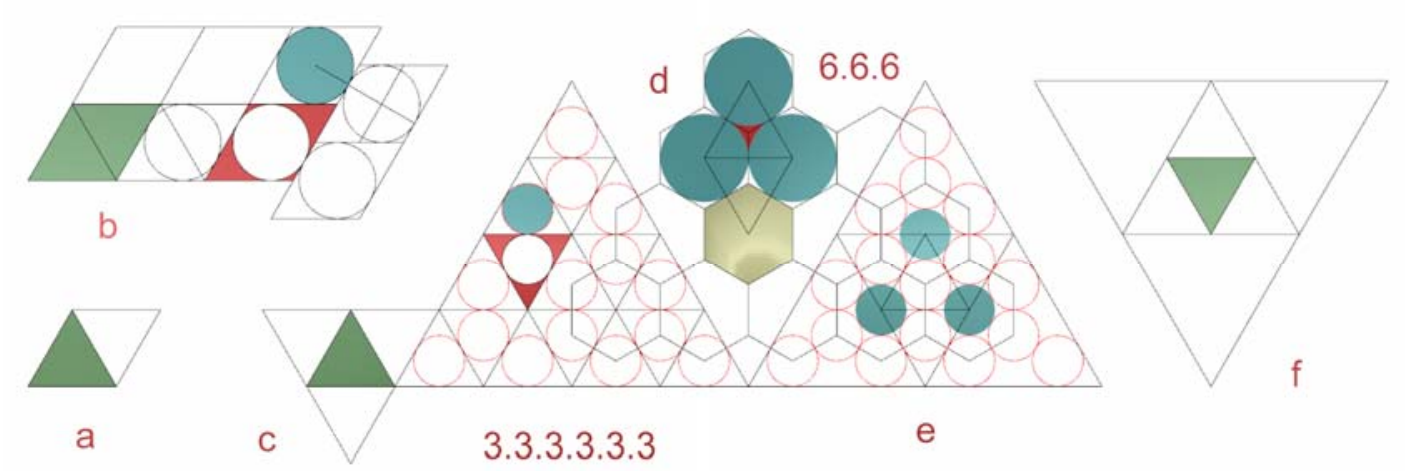

Figure 1

prototile and proceed attaching the same prototile around its edges we may generate this way a regular triangle tessellation $\left(3^{6}\right)$ (fig. 1d). We can see this tiling can get arranging strips with diamonds and then we can attach these rows in columns configuring same regular triangle tilings. Following similar argument and starting from the set of 4 triangles (fig. 1c) we can take this triangle like new prototile and generate a new tiling attaching new prototiles and so on (fig. 1f). This way we can generate a centripetal tessellation where tiles are changing in size but not in shape keeping similarity ratios in 
its growth. This growth can be addressed inward or outward (centripetal or centrifugal) producing a sort of fractal growth.

Another analysis we can make from tilings derivates from building in base of primaries concerned to circles packing analysis resulting of inscribing circles into prototiles. If we divide the surface of inscribed circle between the prototile's surface that is contained, we will get an idea about degree of compactness of tangent inscribed circles packing in a specific tiling. This ratio can be show in $\%$ whether defining it like voids ratio $(\mathrm{H} \%)$ or like compactness ratio $(\mathrm{C} \%=100-\mathrm{H} \%)$ in the same way when engineers test materials porosity. In this case voids ratio of regular triangle tiling would be $39.54 \%$ and compactness ratio $60.46 \%$. In this specific case the circle packing leaves voids which can be filled with circles the same diameter increasing in this way the compactness ratio (fig. 1.e). If we take circle packing of a regular triangle tiling $\left(3^{6}\right)$ and we draw a network connecting circles centers we consequently get a regular hexagon tiling $\left(6^{3}\right)$. In this case the void ratio is only $9.3101 \%$ which shows high degree of compactness this tiling get (fig. 1e). Curiously both two regular tilings among them are dual by means of connecting circles packing centers. This peculiar feature lead to think about procedures in order to getting new tilings starting from others ones known be means of simple connecting of inscribed circles packing centers. The result will be as well tessellation though new tilings do not have to be a tessellation with the same level of regularity or similar features than de provenance tiling. Dual relationships between both tilings are similar which exist between Delaunay (Delaunay, 1934) triangulation and Voronoi diagram. We will see it forward when analyze the uniform dihedral tiling $3^{4} .6$.

Continuing with triangle we are going experiencing attaching triangles by vertex arranging it in equilibrium position making simple reflection (fig. 2a). If we continue from new triangle proceeding in the same way adding triangles by vertices we will back

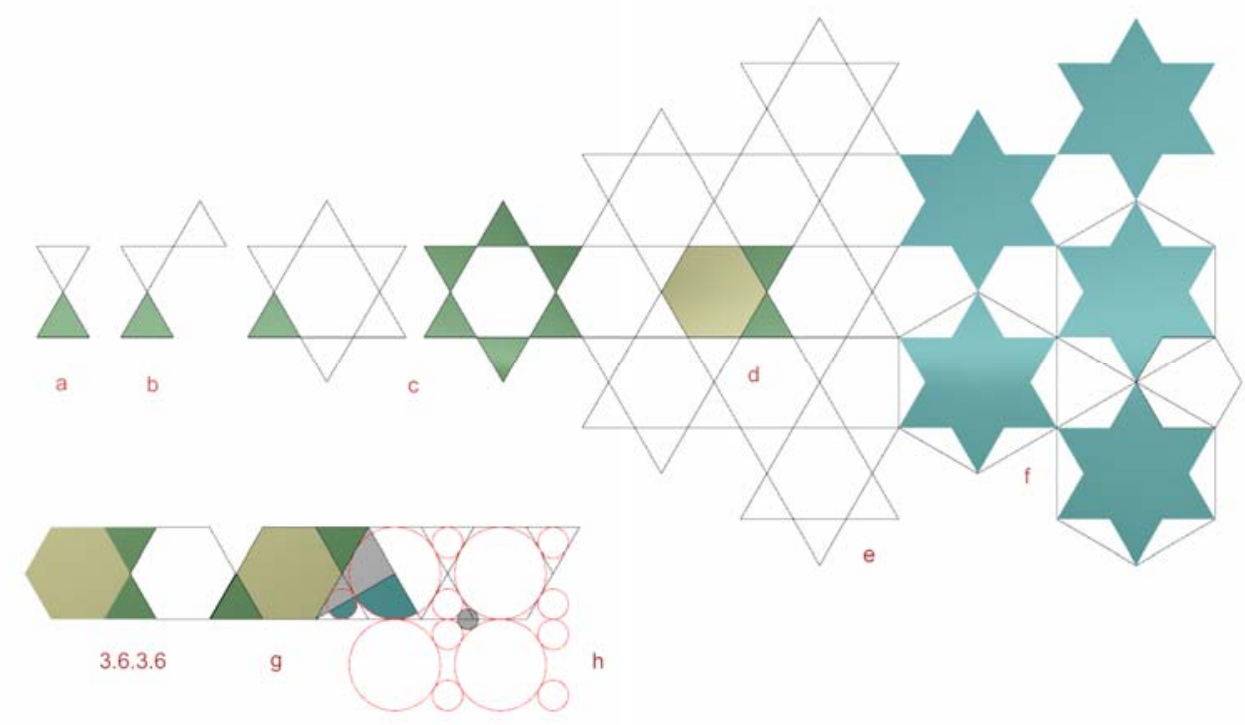

Figure 2 
to the primary triangle. As a result we will get a star composed of a chain with 6 triangles and 1 hexagon inside (fig. 2c). This tiling suggests concepts like figure and background in a tiling. The prototile we have used to experiment covers the plane leaving voids that are filled with other prototile. To improve perception we must use color filling prototiles used to generate tiling. In this way visual contrast is improved. We must not forget that are attempting to get different tilings working as artisans. If we replicate same procedure attaching these star-prototiles by vertices we will get a net of rows and columns which tessellate the plane. This star-prototile is a peculiar cuasiregular concavo polygon known as regular staring polygon. This star can be obtained by means of elongation of a regular convex polygon. Most important in this tiling is not only generate an uniform tiling with triangles and hexagons (3.6.3.6) (fig. 2d) but it also generate an interesting tiling combining 6-stars with hexagons. This idea open the artisans a new world of tilings to use in his ornamental patterns (fig. 2f). On the artisanal side tiles of tiling (3.6.3.6) can be group in diamond shape (fig. 2b) which allows manufacture floor tiles in a fashion easier. The circle packing of this tiling gets a void ratio of $7.9849 \%$, much better tan tiling $6^{3}$.

Starting from the last star-prototile obtained in figure $2 \mathrm{c}$ we can continue adding a ring of triangles attached by its edges getting this way a new group of $n$-gons forming a swastika-like with 6 arms that tessellate the plane (fig. 3). We can add triangles in two different sides of primary triangles thus the swastika can have two different rotation directions which it known as chiral or enantiomorphic tiles (fig. 3aa'). This new figure can be considered whether as a set of prototiles or as a new prototile grouping primary tiles with 18 equals edges. This group of primaries that tessellate the plane we call figure. This figure can be attach in rows an then in columns followings main axis of translational parallelogram which underlines in this tilings. These main axes following network of circles packing of tessellations $3^{6}$ and $6^{3}$. As a result to have this network it can arrange in groups of three figures with 3-rotational symmetry. If we decompose the figure we have primaries triangles and hexagons in a uniform dihedral tiling which tessellate the plane by means of translations. Thus we have rotations and translations in the same tiling. If we want map this tilings with minimal number of colors in such a way the same color do not join each other we will need at least 3 colors because symmetry is 3-rotational. We also can group 3 of these enantiomorphic figures in a new

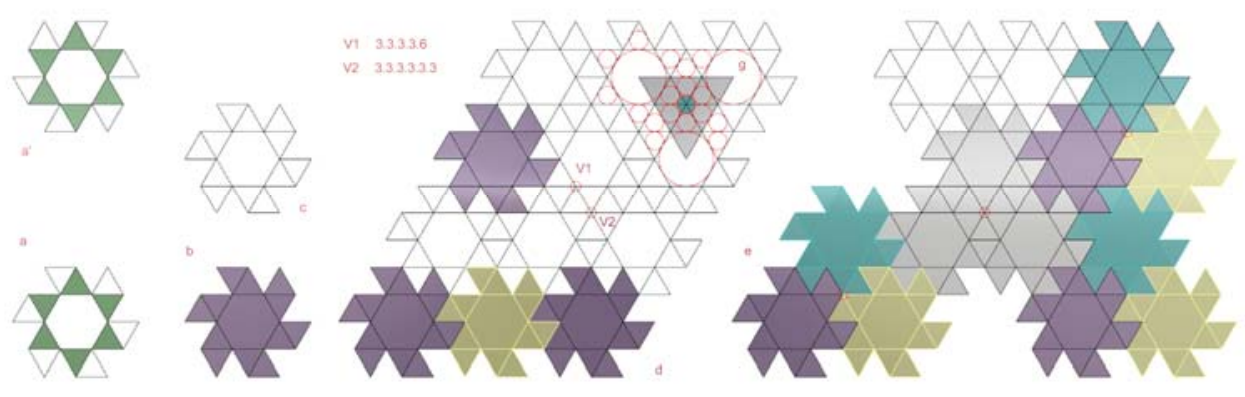


figure which can also tessellate the plane by translations (fig. 3e).

Analyzing this tessellation after decompose this tiling coming back to primaries and its own network with vertices and edges we will can notice two types of vertices. One will be $3^{4} .6$ and another $3^{6}$. Such reflection would lead Krötenheerdt (1969) to consider it would be necessary to establish a range ( $k$-uniform) for different types of vertices into a same tiling, from $k=1$ for uniform tilings to $k=7$, maximum possible range in tilings formed from primaries. If we analyze all the possibilities of regular n-gons concurrence in a vertex we will only find 21 types of possible vertices. $3^{6} / 3^{4} .6 / 3^{3} .4^{2} / 3^{2} .4 .3 .4 /$ $3^{2} .4 .12 / 3.4 .3 .12 / 3^{2} .6^{2} / 3.6 .3 .6 / 3.4^{2} .6 / 3.4 .6 .4 / 3.7 .42 / 3.9 .18 / 3.8 .24 / 3.10 .15 /$ $3.12^{2} / 4^{4} / 4.5 .20 / 4.6 .12 / 4.8^{2} / 5^{2} .10 / 6^{3}$. Otto Krötenheerdt finds all possible $k$ uniform tessellations. For $k=1,11$ cases ( 3 regulars +8 Archimedeans), for $k=2,20$ cases, for $k=3,39$ cases, for $k=4,33$ cases, for $k=5,15$ cases, for $k=6,10$ cases, for $k=7$, 7 cases. For $k$ equal to or greater than 8 does not exist $k$-uniform tessellations. The number of symmetry groups of all possible $k$-uniform tessellations is 17 , curiously the same number of crystallography groups in the plane. We can visualize all these possible $k$-uniform tessellations in a computer application loaded in Internet by Brian Galebach.

Continuing with the same figure star-shaped we before built we can expand this figure adding a new ring of triangles attaching them to configure a hexagonal tile (fig. 4a) which also tessellate the plane (fig. 4b). By using this hexagonal tile as figure we can try different combinations of tiles searching possible intersections or overlaps of tiles (fig. 4c,d). If we combine these figures vertex-to-vertex we get an interesting tiling where it appears regular triangles larger than primary triangles (fig. 4e). Obviously we can divide these big triangles in 4 primary triangles but also we can let be in that fashion and that will allow come in a new field of tilings where tiles or primaries can have similar ones with different sizes. This way would open possibilities to create more tilings built with regular $n$-gons especially in the field of fractals.

Continuing with possible overlaps of these hexagonal figures composed of triangles and hexagons, it exist a particular case of overlapping which gives rise to a uniform tiling, namely, having the same type of vertex in all its knots (fig. 5a). Combination of two
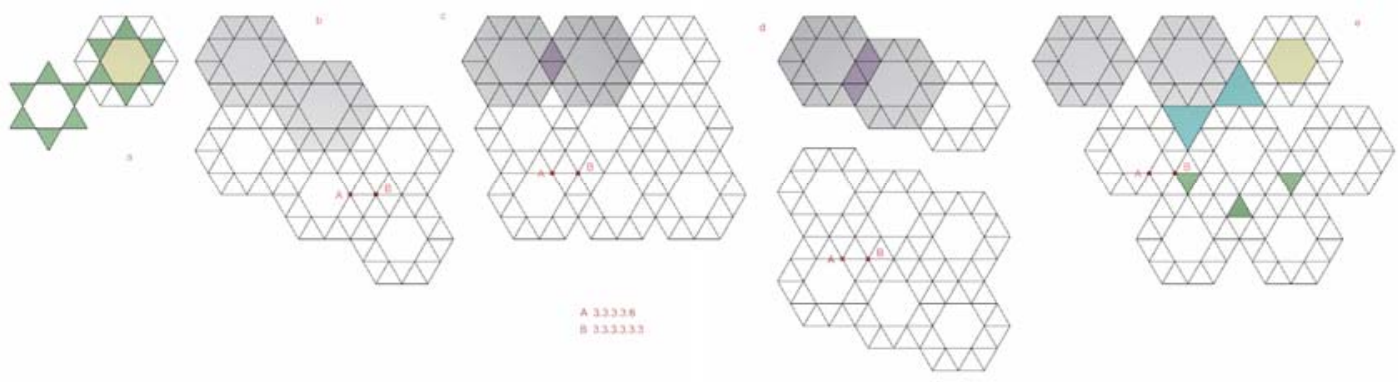
figures sharing 4 triangles is different from fig. 4.b, but in this case 2 figures group with other figure (3 figures) conforming a set in 3-rotational symmetry. This new set has translational symmetry when generate tiling which have property to be uniform tiling type of $3^{4} .6$ (fig. $5 b$ ).

Basing on this specific tiling we will establish a set of concepts to better understand this world under artisans and designers point of view. Tessellation can be understood as a set of edges and vertices shaping network but also as a set of tiles attached edge-toedge. For mathematicians is very important analyze features and properties of tiles geometry and properties of vertices and knots of the network as well symmetry

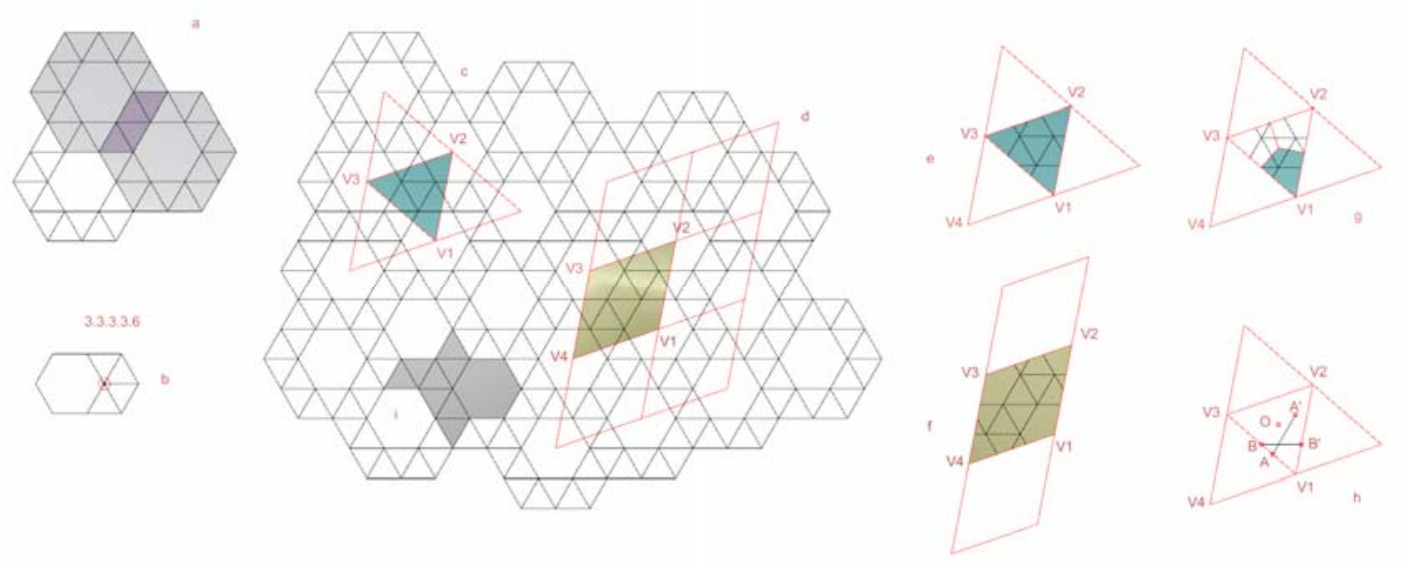

Figure 5

properties. For artisans and designers it is not so important. They search for simple methods to make artistic creations based on tilings.

All information of this tiling can be in a simple triangle V1V2V3 (fig. 5c) by means of connecting centers of 3 figures. This information can even be more synthesized up to reduce information to a minimum which can generate utter tiling from this dim piece of information by means of all sorts of symmetry operations. Here we can consider if this minimum piece must be a fragment from network or a piece containing entire $n$-gontiles capable of regenerating complete tiling. First case, considering tiling as network we can reduce triangle V1V2V3 a minimum of 2 segments from which it can generate complete tiling. This minimum information consist of segments we call seed-graph. In this particular case the seed-graph consists of only 2 lines AA', BB' and a point $\mathrm{O}$ as reference point from which generate triangle V1V2V3 and then complete tiling be means of symmetry operations (fig. 5.h). However, if we want the minimum information be contained in a tile which tessellate the plane we use a seed-tile which 
contain segments but has properties of $n$-gon like edges an vertices (fig. $5 \mathrm{~g}$ ). In this case the seed-tile is kite-shaped and if we make 3-rotations of this kite round his obtuse angle we get a triangle containing necessary segments to generate complete tiling. This set of information forming a triangle is a primary-tile (fig. 5e).

If we want reduce information in a tile which can generate tiling by means of only translations we must group the minimum information in a parallelogram V1V2V3V4 we call parallelogram-tile (fig. 5f). The information contains segments of a network but if we want reduce the minimum information in such a way the parallelogram contain only enter $n$-gons, prototiles or primaries then we call figure this group capable of generate complete tiling by mere translations (fig. 5i). This type of templates has been used by artisans around the world through History and it is even being nowadays used by craftsmen in Morocco and other countries. The parallelogram-figure does not have to be unique but it must be the minimum. In other words, it must contain a minimum of $n$-gon-tiles to be capable of generating tiling by just translations. Most interesting in this approach is that it can be more than one figures which fulfill requirements of generating tiling by only translations and contain minimum entire $n$-gon-tiles (fig. 6b,c) though it allows rotational symmetry operations (fig. 6e). If there is more than one possible figure it is necessary theses ones have the same number of primaries or n-gono-tiles (in this case: 8 triangles and 1 hexagon). In case of a figure be parallelogram-shaped we call this one parallelogram-figure. One question we can ask is: ¿how many translational figures can exists in a specific tiling and what conditions must have? Those are sorts of questions interest to mathematicians but not so much to artisans.

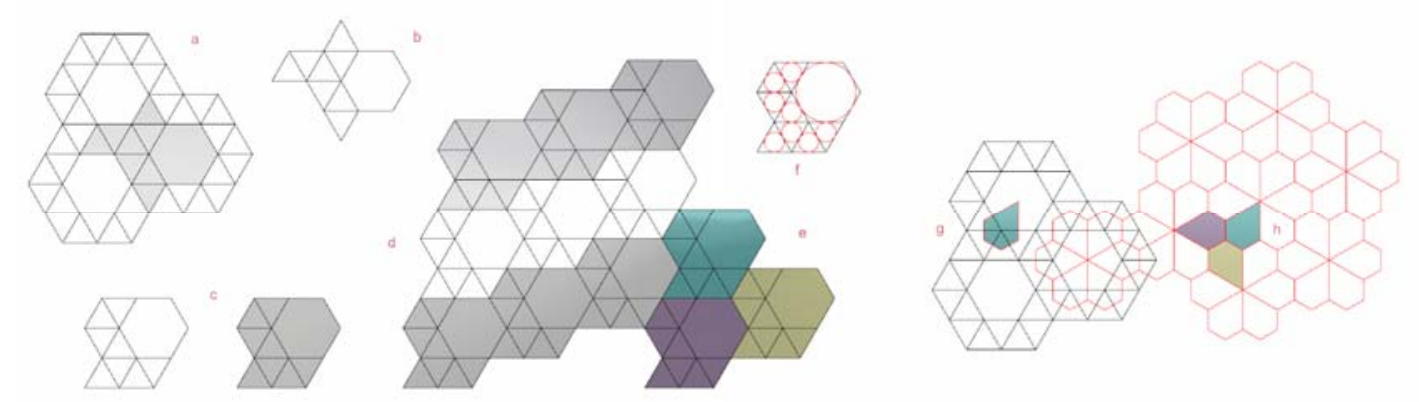

Figure 6

Coming back to artisan point of view we can watch tiles shape and tiles colors. If an artisan wants color tiles or figures he encounters a problem that mathematicians have 
resolve. ¿how many different colors it need to color a tiling without put the same color edge to edge? Most of tilings have the answer 2 or 3 . The answer is 2 when symmetry is only translational or 3 when tiling have 3-rotational symmetry. We can generalize it saying: if the seed-tile is parallelogram-shaped (square or diamond) then tiling only needs 2 colors and if the seed-tile is triangle-shaped it is needs 3 colors.

Artisans had empirically discovered these answers long ago. Other sort o things interest to artisans are how transform or handle a specific tiling to transform it in another tiling or in another ornamental pattern. One possible method is create a network by connecting $n$-gon centers from a tiling getting a new tiling (fig. $6 \mathrm{~g}-\mathrm{h}$ ). Another option for artisans is forms a group of $n$-gons or primaries and configures a new figure though do not be translational or do not generate tiling itself. We call these figures free figures and we can group different free figures to form a figure which be translational and could tessellate the plane. Methods like those allow artisans and designers create different ornamental patterns. In this sense and from the point of view of visual perception we can make up a tiling to stand the silhouette out like a picture when we can perceive figure and background that improve perception and give a sense of depth and relief. To

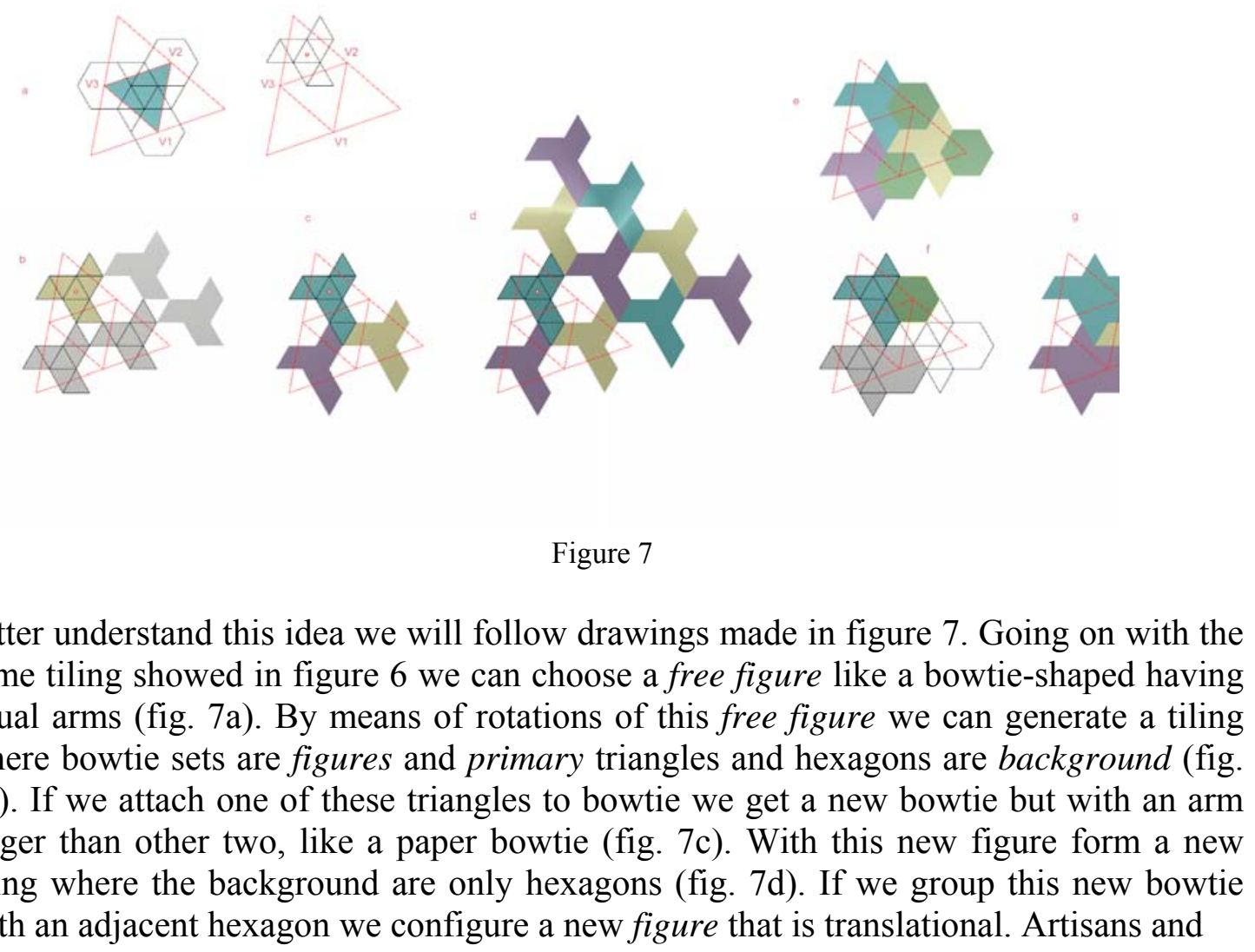

better understand this idea we will follow drawings made in figure 7 . Going on with the same tiling showed in figure 6 we can choose a free figure like a bowtie-shaped having equal arms (fig. 7a). By means of rotations of this free figure we can generate a tiling where bowtie sets are figures and primary triangles and hexagons are background (fig. $7 b$ ). If we attach one of these triangles to bowtie we get a new bowtie but with an arm larger than other two, like a paper bowtie (fig. 7c). With this new figure form a new tiling where the background are only hexagons (fig. 7d). If we group this new bowtie with an adjacent hexagon we configure a new figure that is translational. Artisans and 


\section{,}
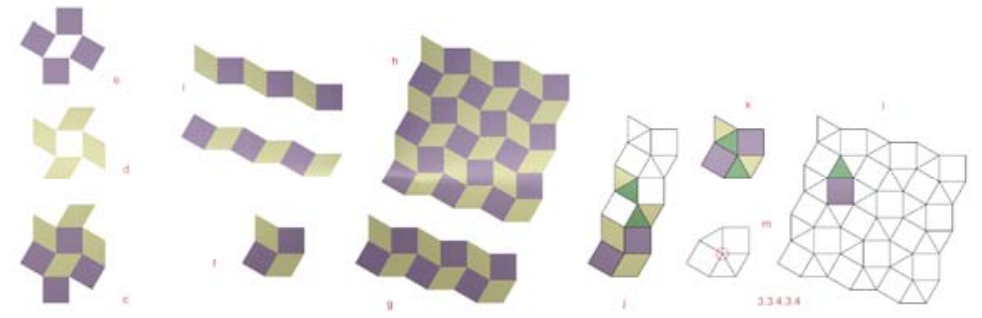

Figure 8

graphic designers like M.S.C. Escher (1898-1972) (Escher, 1971) used basic tilings as references to built onto them different arrangements to elude follow excessive rigidity of tiling patterns. Of course there are much more methods to transform previous tilings to achieve artistic or ornamental patterns like make transformations in tile edges as Escher used to do. Continuing with same strategy of attaching primaries to get tilings, in the same fashion artisans would make, we will go on using triangle as primary. We join two triangles edge-to-edge and get a diamond. Then we use diamond as prototile making rows and columns getting well-known checkerboard but in this case diamondshaped because the angle is different to $\pi / 2$ (fig. $8 \mathrm{a}$ ). Using the same prototile we can change tile's orientation and try to attach by vertex (fig. 8b). This arrangement does not allow fulfill the gap with diamonds but squares. In this way we generate a new tiling which can be perceived like square over diamonds or diamonds over squares (fig. 8c-de). This type of tiling has another interesting property: it may be split in strips though these strips are not sliding because its edge is not a straight line (fig. 8i). The minimum translational-figure is composed by 4 prototiles ( 2 squares and 2 diamonds) but these prototiles are not translational between them because both of them have different orientations (fig. 8f). That is to say: there are 4 translational prototiles whereas only 2 of them are congruent. If we go back and separate diamonds in its primary triangles we will get an interesting uniform-tiling type of 3.3.4.3.4. However the new tiling needs 3 colors to map the tiling whereas square and diamonds tessellations only need 2 colors (fig. 8i-j-k-m).

Hitherto we have been experimenting attaching primary triangles to generate tilings. Continuing same artisanal procedure we should experiment combining different primaries between them and watch these experiments in order to discover new tilings or patterns. We will start attaching a square to primary triangle edges as we can see in figure $9 \mathrm{a}$. 
After attaching first ring with squares we watch angles squares leaves between them to know what type of $n$-gon could fulfill it gap. In this case hexagon is a primary which fulfill this gap between squares forming a second ring of prototiles. Connecting hexagons centers we get a triangular seed-tile which tessellates the plane forming a uniform-tiling type of 3.4.6.4 (fig. 9b-c). We can also see this tiling can be split in strips though not sliding ones but complementary strips (fig. 9d). When we attaching both complementary strips can see a translational figure composed by a set or primaries: $2 \mathrm{P} 6+3 \mathrm{P} 4+2 \mathrm{P} 3$ ( 2 hexagons +3 squares +2 triangles) (fig. 9e). It is necessary 3 colors to map this tiling. Because this seed-tile is triangle-shaped we need group 2 seed-tiles by means of a rotation of $180^{\circ}$ to form a parallelogram-tile (fig. $9 \mathrm{~b}$ ).

If we take the second ring configuration and test exterior angles we will verify regular 12-gon fulfill the gap. Regular 12-gons will form a third ring (fig. 10b). Connecting 12gons centers we get a triangular seed-tile which tessellates the plane configuring a 2uniform-tiling with 2 different vertices: 3.4.6.4 and 4.12 .6 (fig. 10c-d). It is very interesting remarks this tiling have 2 different arrangements, one with a triangle seedtile and another with a square seed-tile keeping same type of vertices (fig. 10e-f). These two tilings have in common 2 primary strips: one strip composed by 12-gons and
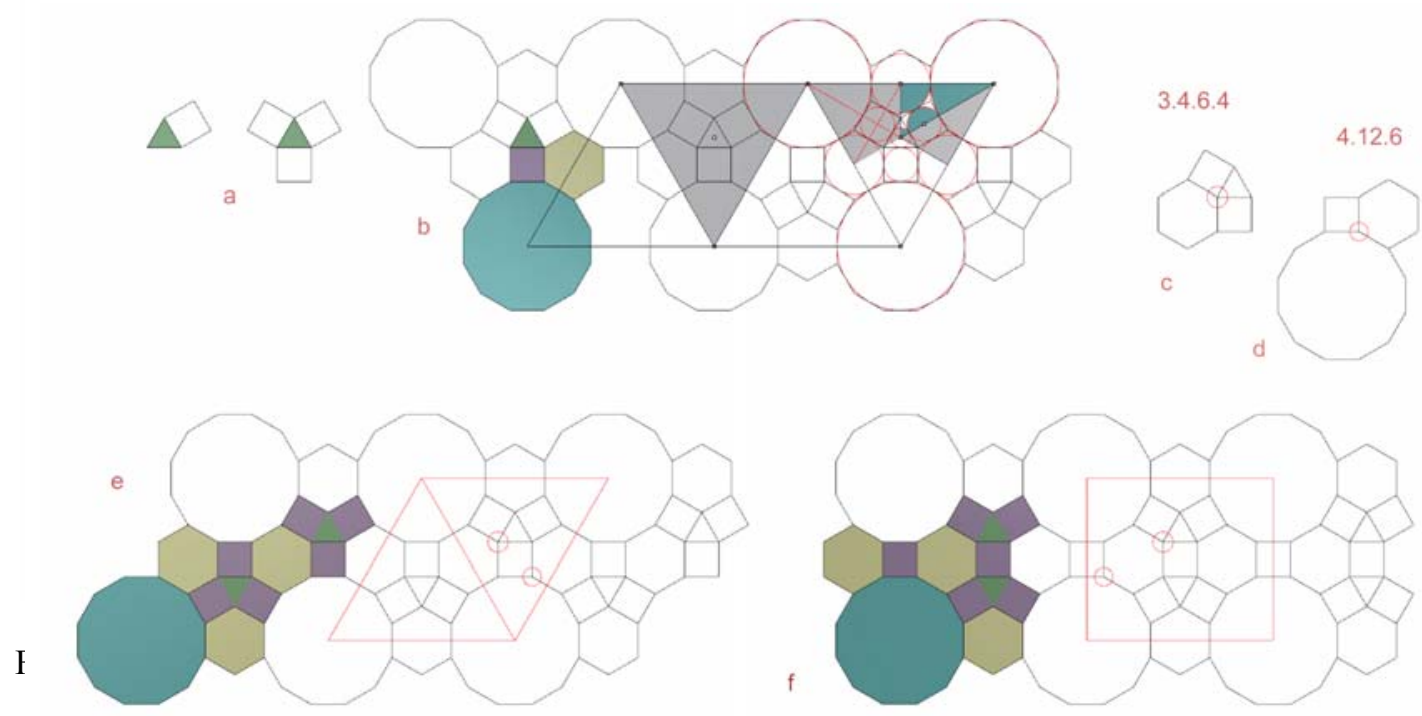

Figure 10

hexagons (1P12+1P6) and another strip composed by hexagon and squares (1P6+1P4). Overlaps of these strips are cuasi-congruent leaving voids can be fulfilled with a set of primaries $(1 \mathrm{P} 4+1 \mathrm{P} 3+1 \mathrm{P} 4)$. The translational-figure must contain at least following primaries: 1P12+3P $6+6 \mathrm{P} 4+2 \mathrm{P} 3$ (fig. 10f). 


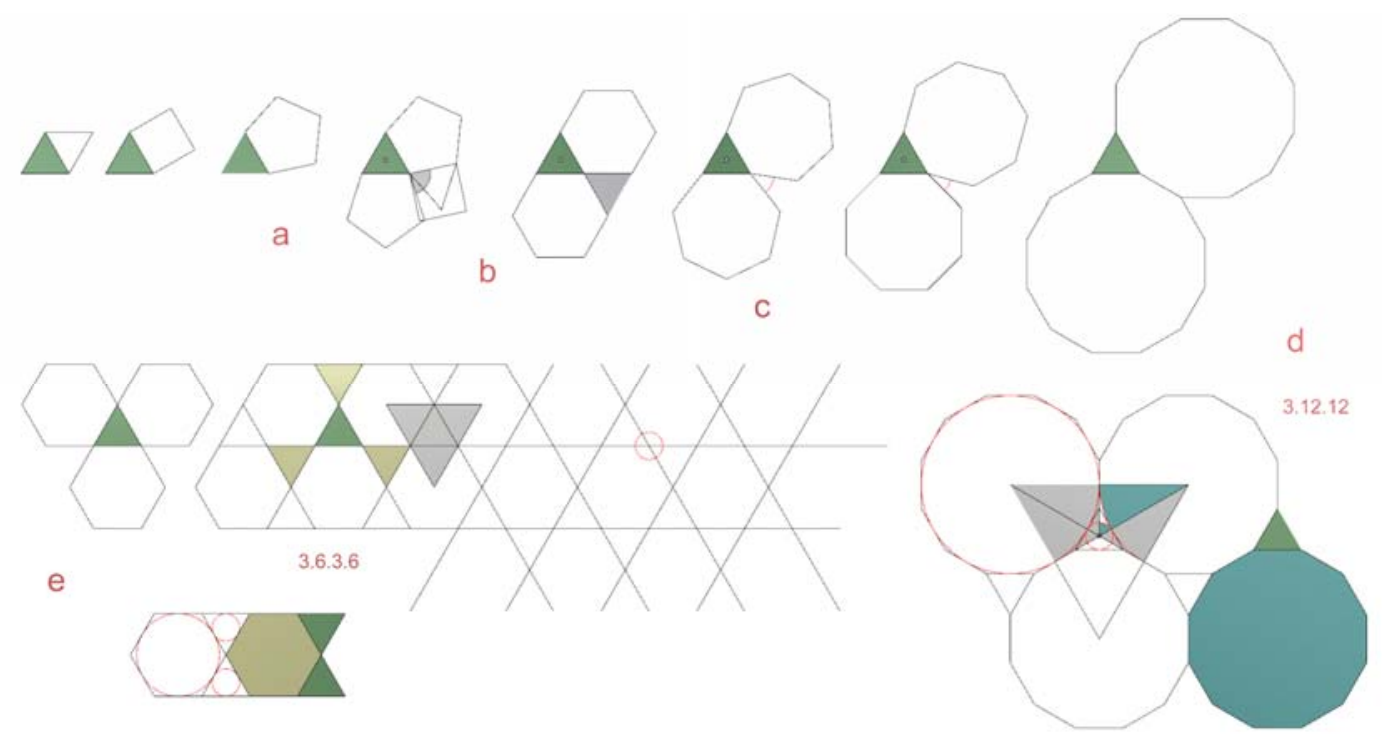

Figure 11

Following same procedure we must go experimenting on attaching primaries increasing number of edges. After attaching square we should add pentagons and regard what sort of angles left between them (fig. 11a). In this case is not possible to find a regular n-gon that fit de gap. Next n-gon is hexagon and now gap is fitted by triangles (fig. 11b). If we going on experimenting $n$-gons with more sides $(7,8,9 \ldots)$ the gaps are not fitted up to regular 12-gon (fig. 11d). In case of hexagons first ring we can get a uniform-tiling type of 3.6.3.6 (fig. 11e) which already got before (fig.2). This is one of the tree tilings fulfilling requirement of forming lattices with straight families: $\left(4^{4}\right),\left(3^{6}\right)$ and (3.6.3.6). Tilings $\left(3^{6}\right)$ and (3.6.3.6) have same straight families -same angles $(\pi / 3)$ between families- but have different relative positions. Tiling $\left(4^{4}\right)$ has orthogonal lattice with angles of $\pi / 2$. In case of regular 12-gons we can form a first ring getting in this way a uniform-tiling type of $3.12^{2}$ from 2 primaries, triangle seed-tile and simple translational figure (P12+P3) (fig. 11d). This tiling has best compactness ratio of all uniform tessellations $(95.03 \%)$.

\subsection{Primary-square}

After exhausting possibilities of experimenting with basis primary triangle now plays experiment with squares following same procedure. We will put square tile like basis and forming first rings with n-gons with more number of sides (P3, P4, P5 ...) (fig. 12a). We will start forming first ring with triangles (fig. 12b). Angles between triangles are fitted with regular 12-gons which form second ring without gap and we have a new tiling. This tiling has square seed-tile and 2 vertices: $3.12^{2}$ and 3.4.3.12 (fig. 12e-d). Going on with procedure now plays to form first ring with squares. In this case result is 
already known giving tiling $4^{4}$. Following procedure we experiment with next n-gons P5, P6 and P7 but angles between first ring n-gons do not fulfill requirements to fit other regular n-gons up to form first ring with octagons. In this case we will get a new uniform-tiling type of 4.8 .8 (fig. 12b). From this n-gon (P8) procedure enters in dead end because next n-gon (P9) is overlapped in first ring.

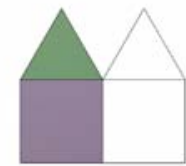

a

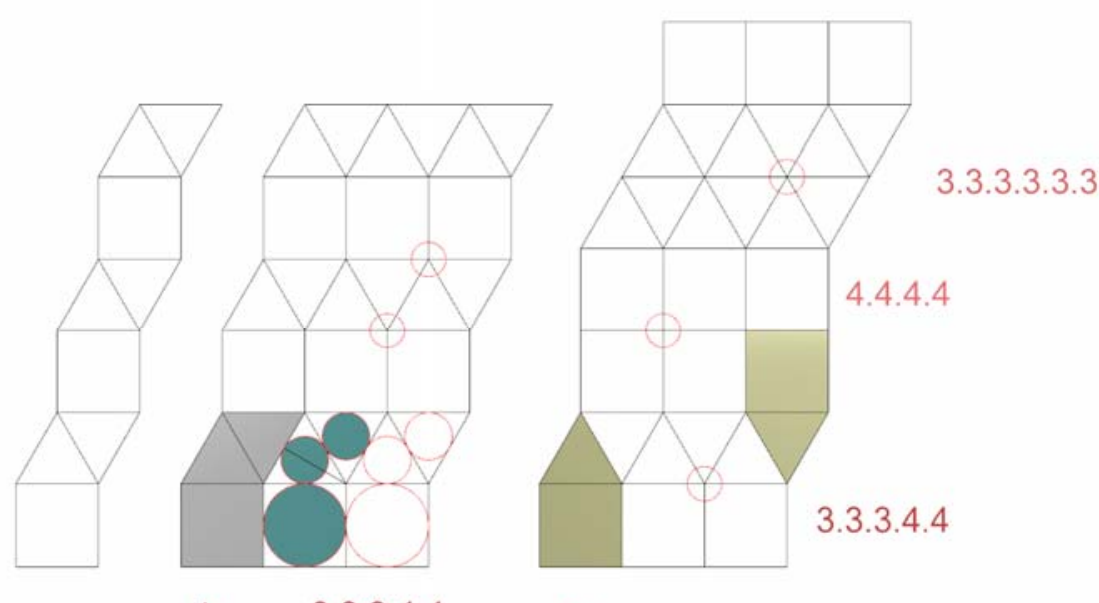

b $\quad 3.3 .3 .4 .4$

C

Figure 14

Hitherto we have made procedure forming rings around primaries but another method is possible in some cases. We are talking about make strips with primaries and then add strips forming columns fitting gaps and tiling the plane. This is case of figure 14 where we form strips with squares and triangles. As result of adding strips we get a uniformtiling type of $3^{3} \cdot 4^{2}$ (fig. $14 \mathrm{~b}$ ). If we randomly combine rows of squares or triangles result can be tilings having 3 possible different vertices type of $\left(3^{3} \cdot 4^{2}\right),\left(4^{4}\right)$ and $\left(3^{6}\right)$ (fig. 14c). This fashion to get tiling from strips we call strip-tiling. Until now all tiling shown are edge-to-edge tilings but we can open other possibilities to configure tilings if we allow side-vertex joins as we show in figure 15 though many properties are lost. Actually in this sort of tilings tiles are more important than vertices or knots network. Mathematically these tilings lose many properties but from artisans point of view combinatorial possibilities are increased. 


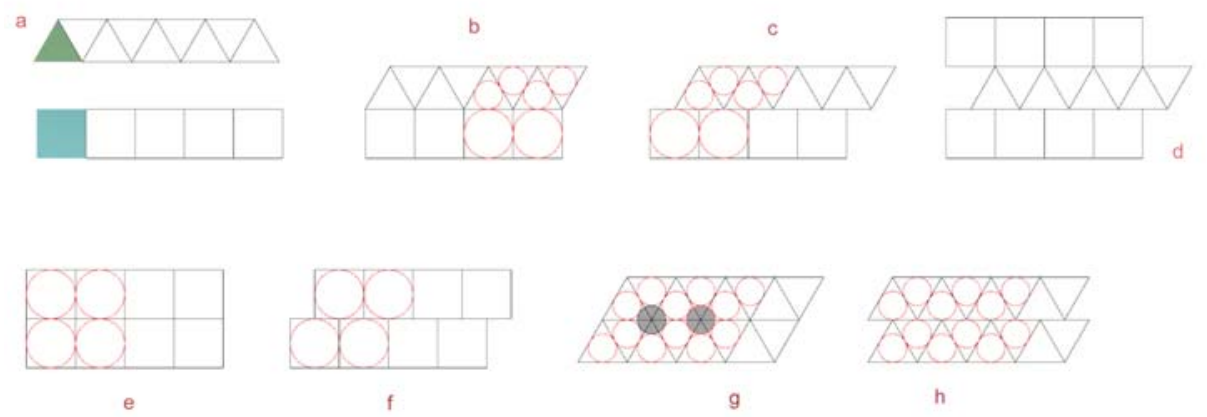

Figure 15

\subsection{Primary-pentagon}

Now we will play with pentagon like basis primary. We will start attaching pentagons by side because already tested triangles and squares above. In figure 16a we show how to generate a ring of pentagons and how it can combine with other one by means of decagons and a cuasi-regular hexagon (equal edges but 2 different angles) obtained from fusion of two pentagons. This way we get a interesting tilings with a parallelogram-seed-tile although is not a uniform-tiling (fig. 16b). Nonetheless this tiling is very interesting because tessellation is shaped with equal edges (iso-edge) and also has same type of vertex in its network $\left(5^{5} .10\right)$ as usual in uniform-tilings. In case of starting attaching pentagons by vertex we can get a chain of pentagons where interior polygon is a regular star-gon having 20 sides (S20) (fig. 16c). If we make reflection with all chain pentagons we will get one more than curious graph known as 10 -arms Islamic star pattern especially used by medieval Persian culture. This type of stars has

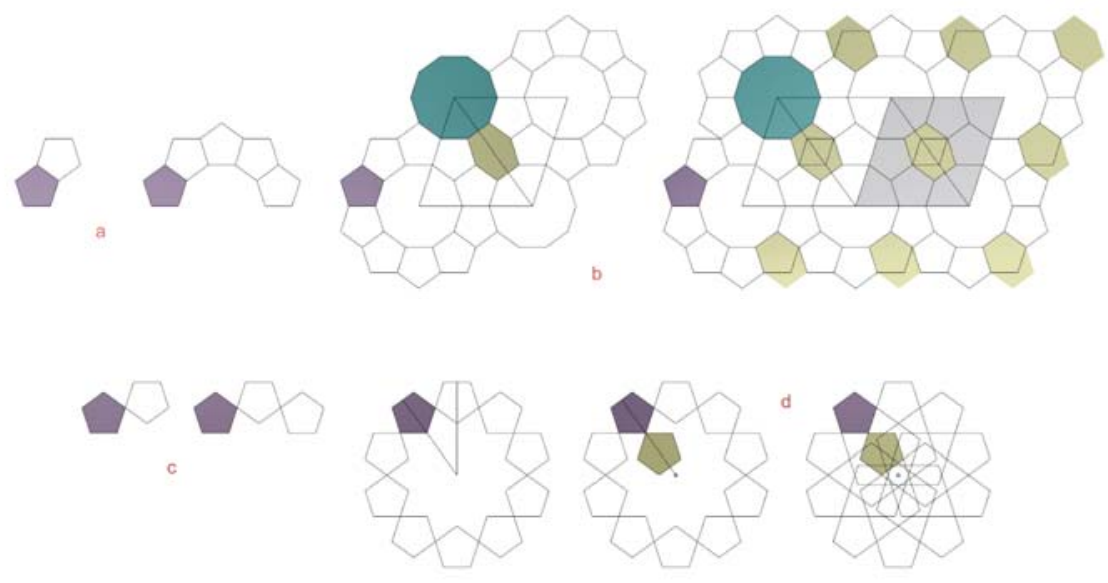

Figure 16 
been very important in Islamic culture through History and one detailed analysis leave the limits of this paper.

\subsection{Primary-hexagon}

Following procedure we will play with hexagon as basis primary. Attaching triangles we will get a well-know uniform-tiling type of 3.6.3.6 with two possible translational figures (fig. 17a-b-c). If we try attaching squares and forming a ring gaps between squares can be fitted with triangles configures a first ring without gaps. This set would be rise to one tessellation 3.4.6.4 we already analyzed in figure 10. Instead of attaching full squares ring we eliminate three of them as shown in figure $17 \mathrm{e}$, the new gap can be fitted with regular 12-gons closing first ring and getting a new tiling form a triangle seed-tile. Tiling is uniform type of 4.6 .12 and his figure has $1 \mathrm{P} 12+3 \mathrm{P} 4+2 \mathrm{P} 6$ (fig. 17f). This tiling is the same above we got in figure 10e-f.

\subsection{Others n-gons primaries}

To follow utterly procedure we must go putting nest primaries increasing number of sides (P7, P8, P9 ...) up to exhaust all possibilities getting dead ends or rediscover already known tilings as above we did. In fact summarizing our experimental method we can check we already have rediscover not only the three regular tilings (36 / 44 / 63) configured with only one primary but the 8 semi-regular, Archimedeans, or uniform-

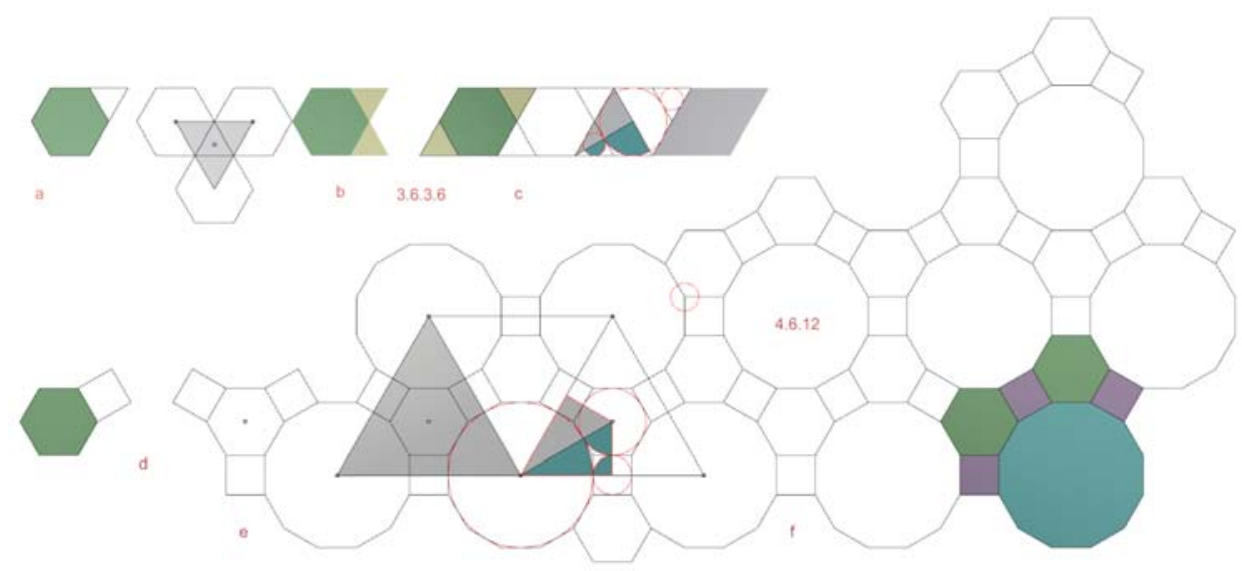

Figure 17

tilings formed with more than one primary (3.6.3.6 / 3.4.6.4 / 3.122 / 32.4.3.4 / 33.42 / $34.6 / 4.6 .12 / 4.82)$. By the way we also have got other interesting tiling from artisans and designers point of view. 
Unlike to artisans and designers mathematicians would wonder how many vertices are possible using primaries as tiles to form tilings. Krötenheerdt (1969) found 21 possible cases. The key is to assume the sum of all concurrent angles in vertex must be equal to $2 \pi$ and that only angels of regular n-gons are possible. In this way all possibilities of discover more uniform-tilings is closed. In order to advance discovering new tilings it is necessary losing regularity. If we restrict tiling using only monohedrals compositions built with an only one primary there are only 3 regular tilings. If we lose regularity conditions and we allow more than one primary to form tiling keeping periodicity in vertices there are 8 uniform-tilings all of them 2-hedrals but tiling 4.6 .12 that is 3 hedral. If we go on losing regularity allowing different types of vertex in same tiling possibilities would rises up to 135 tilings called k-uniform by Krötenheerdt (1969). From now no more tilings shaped from regular convex n-gons would be possible however a large field of tilings would be possible keeping same edge but allowing regularity losing in n-gons angles i.e. isosceles triangle or diamonds). This type of ngons we call cuasi-regular $n$-gons when only have 2 different angles $(\alpha 2)$ because they are closest to utter regularity. In this field we must notice star-n-gons which have inscribed and circumscribed circles touching all vertices. That type of cuasi-regular ngons are very important in tilings based on pentagon-decagon networks and other type of interesting tiling from ornamental pattern point of view.

\section{APERIODIC TILINGS AND OTHER TILINGS}

When a tiling it is not possible to generate from a seed-tile by means of making systematic symmetry operations we call these ones aperiodic-tilings. Discovering of that type of tilings took place into medieval Islamic culture around X and XI centuries in cities like Bagdad and Isfahan. These new tessellation were arose from pentagondecagon Islamic patterns. Some authors (Hogendisjk 2012, Özdural, 1995 and 2000) suggest geometricians or mathematicians like Omar Khayyam (1048-1131) could have helped to design some Islamic patterns in Isfahan where this medieval scientific resided. We know ornamental pattern's artisans that Age consulted mathematicians in order to solve specific geometrical problems they had experimenting with its ornamental patterns (Abu'l Wafa, ca. 990). Most of these patterns are based on pentagon-decagon but some is also based on heptagon though the drawing of this regular n-gon was known it was not geometrically right (Abu'l Wafa, 10th-c, and Omar Khayyam, 1048-1131) though artisans and ornamental designers do not worry about geometrical perfection but beautiful and complexity. 
In the beginning of $\mathrm{XX}$ century, Hankin start exploring secrets that type of Persian pattern looking for guidelines to generate them (Hankin, 1905 and 1925). At the end of XX century interest in this patterns is increased and Penrose (1974) goes on exploring aperiodic tessellations based on pentagon-decagon Persian patterns and he discover new aperiodic-tilings which have similarities in Physic and Nature fields. Recently there has been a renewed interest on that type of Persian Islamic star patterns from different points of view especially for its relationships with cuasi-crystals. (Rull Pérez,1987), (Bonner, 2003), (Lu and Steinhardt, 2007a y b), (Saltzman, 2008), (Cromwell, 2008), (Prange, 2009), (Cromwell and Beltrami, 2011), (Hogendisjk, 2012) and (Bier, C. (2012). Aside from ornamental Islamic star pattern we can regard in historic Persian architecture there are very interesting documents known as Topkapi Scrolls (Necipoglu, 1995) dated to the XVI century (Topkapi Palace, Istanbul, Turkey). It can see many beautiful and complex drawings nonetheless there are not explanations about what geometric procedures were made. Based on one of these scrolls we show a Islamic star pattern where it has overlapped circumscribed circles to form tessellation basis of this ornamental pattern (fig. 18). We can notice whereas A, B, C circles circumscribe star-ngons complying with tangency requirements, circle $\mathrm{D}$ has slight error in circles tangency. That sight error does not worry so much to artisans but has very interest to
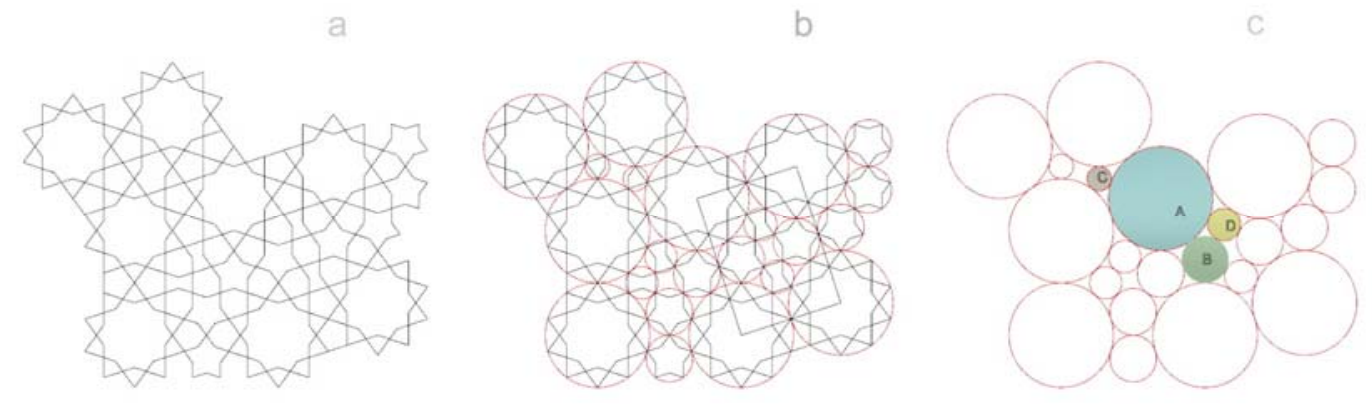

Figure 18

mathematicians especially when they analyze relationship between tessellations and circles packing (fig. 18b-c). In this case the right solution can be solved from Apollonius's problem. These slights errors are common in many Islamic star patterns and have interest to mathematicians though we have no room in this paper to better explain this topic. However we want remark this Persian Islamic star pattern is composed by periodical sets and other aperiodic ones. In figure 18a we can regard set of 4 10-stars shaping translational parallelogram-tiles and other set of 410 -stars shaping translational squaring-tiles both combining with free parts to configure an aperiodictiling. 
Tools use by artisans like ruler and compass involved circles packing and tilings through History. We will take this question up in further publication from artisan point of view (Fernnande-Cabo, 2014).

This paper restricts to show what fashion could discover regular and Archimedean tessellations by means of simple and experimental methods. Other tiling types developed from not regular n-gons, rotational and spiral tilings (Voderberg, 1937) or hyperbolic tilings have been left out from this analysis.

\section{SOME COMMENTS ON TESSELLATIONS IN SCIENCTIFIC FIELD}

Archimedes (c.287 BC-c. 212 BC) started tessellations research in specific scientific field but interest decays between scientists and are mainly artisan, roman artisans above all, who develop tilings in his ornamental mosaics. In medieval Islamic world it can notice are Islamic artisans who keep interest though History and who begin approaching among both artisan and scientist worlds (Örduzal, 1995, 2000) but it is in Italian Renaissance when scientific world increase interest in tessellations and regularity of forms and volumes (Luca Paccioli,1445-1517, De Divina Proporctione). Later authors like Johannes Kepler (1571-1630) (Harmonici Mundi, 1619) take this topic up. The issue remained more or less slipped long time because this topic seemed to be mathematically trivial (Hilbert, 1862-1943) or getting a dead. In the twentieth century interest in this topic revives especially focusing analysis on aperiodic tiling and cuasycrystals both of them inspired on Persian Islamic star patterns. From artisan and artistic world, Escher awake mathematicians interest and not analytical geometry have revived interest again by means of some scientists like Coxeter (1907-2003) who was a friend of Escher. From the 60's other mathematicians make important contributions in both scientist field (Krötenheerdt, 1969, Grünbaum and Shephard, 1987) and applications in industry and arts (Horne, 2000).

Concern for mathematical proofs on cover the plane with regular n-gons comes from a very old time. There is a very simple proof that only regular triangle, square and hexagon configure regular tilings. In each tessellation vertex the sum of the angles must to be $2 \pi$ and polygon angles must be $2 \pi$ integer dividers as well. Minimum n-gon that fulfill requirements to cover the plane is the triangle $\left(60^{\circ} \times 6=360^{\circ}\right)$. Next n-gon is the square that also fulfill requirements $\left(90^{\circ} \times 4=360^{\circ}\right)$. However next n-gon, the pentagon does not meet the condition because its angle $\left(108^{\circ}\right)$ it is not integer divisor of $360^{\circ}$ and thus does not tile the plane. Next n-gon is the hexagon that does tiles $\left(120^{\circ} \times 3=360^{\circ}\right)$. But from hexagon all polygons with more sides have a greater internal angle bigger than $120^{\circ}$ hence cannot meet the conditions. At least the angle must be multiplied by 3 because it must gather at least 3 polygons at a vertex of the tessellation and therefore does not tessellate the plane. Actually this proof is very similar to the argument attributed to Theaetetus (Athens, c. 417-369 B.C.) who proofs that can only exist 5 regular polyhedra known as Platonic solids (Euclid, c. 325-c.265 BC, The Elements, 
Book XIII). A more technical proof from mathematical point of view can be found in Coxeter (Coxeter, 1961). Coxeter use Schläfli nomenclature (p,q) make following equality: $(1-2 / \mathrm{p}) \pi=2 \pi / \mathrm{q}$. The first term shows interior angle value of a p-gon and the second shows the number of p-gons meeting at a vertex of tessellation. The importance of these three regular tilings can better be understood when it is expressed in terms of symmetry groups. Coxeter himself recalls Barlow's proof (1845-1934) (Coxeter, 1961): the only possible cyclic subgroups are C2, C3, C4 and C6. That is to say that the only possible period in a rotational symmetry operation of a network or lattice are 2, 3, 4 and 6 . Numbers 3,4 , and 6 , obviously correspond to the triangle, square and hexagon while number 2 would correspond to a hypothetical two-sided polygon and of course the number 1 would be the identity.

\section{CONCLUSIONS}

Through this limited paper we have tried to show how artisans and designers could have discovered all 3 regular tilings and 8 uniform-tilings as well as many interesting others ones using very simple methods of experimentation by means of material clay or wood tiles. Method would be merely experimental combining possibilities and layout tools would be string, chalk, punch, ruler and compass (Sutton, 2009). Artisans never tried to develop mathematic science bur they were aware certain geometric challenges they found when working in its works. It should be here recalled that the artisans were the main authors of the practical and experimental development of tilings making its ornamental patterns before some mathematician configure this scientific field. The same happened when Islamic artisans experimented with symmetries of geometric figures. In only one building, the Alhambra in Granada, it can find the 17 symmetry plane groups long before mathematicians had developed the theory of groups and proof the impossibility that there could be more groups that have already been discovered by Islamic craftsmen in his medieval Age. While artisans have never tried to invade the scientific field we must recognize their contribution as pioneers opening and exploring certain territories after scientists finally settled and assumed as their own.

\section{ACKNOWLEDGEMENTS}

I especially want to thank revision work and mathematical assistance by teachers Antonio Casas, Eugenia Rosado, Sonia Rueda and Department of Mathematics, School of Architecture, Technical University of Madrid. 


\section{REFERENCES}

Bier, C. (2012). The Decagonal Tomb Tower at Maragha and Its Architectural Context: Lines of Mathematical Thought, Nexus Network Journal, Vol.14, No. 2, 2012, pp. 251273.

Bonner, J. (2003). Three traditions of self-similarity in fourteenth and fifteenth century Islamic geometric ornament. Proc. ISAMA/Bridges: Mathematical Connections in Art, Music and Science, (Granada, 2003), R. Sarhangi and N. Friedman, pp. 1-12.

Coxeter, H.S.M. (1961). Introduction to Geometry. John Wiley and Sons, Inc. USA.

Cromwell, P.R. (2008). The Search for Quasi-Periodicity in Islamic 5-fold Ornament. The Mathematical Intelligencer, Springer Science+Business Media, LLC.

Cromwell, R. P. and Beltrami, E. (2011). The Whirling Kites of Isfahan: Geometric Variations on a Theme. Mathematical Intelligencer 33 (2011), 84-93.

Cundy, M. and Rollett, A.P. (1951). Mathematical models. Clarendon Press, Oxford.

Delone (Delaunay), B.N. (1934). Sur la sphere vide. A la mémoire de Georges Voronoi. Izvestia Akademii Nauk SSSR, Otdelenie Matematicheskikh i Estestvennykh Nauk (Bulletin of Academy of Sciences of the USSR), 7, págs. 793-800.

Escher, M.C. (1971). The World of M.C. Escher, J.L. Locher, ed. Abrams, New York.

Fernández-Cabo, M.C. (2014). The search for regularity in ornamental geometry I: Tangents Circles in the plane and in single curvature. (to appear).

Galebach, B. http://probabilitysports.com/tilings.html

Gardner, M. (1989). Penrose Tiles to Trapdoor Ciphers. W.H. Freeman and Company.

Gardner, M. (1990). Mosaicos de Penrose y escotillas cifradas. Labor, Barcelona

Grünbaum, B. and Shephard, G.C. (1987). Tilings and Patterns. W.H. Freeman, New York.

Hankin, E. H. (1905). On some discoveries of the methods of design employed in Mohammedan art, J. Society of Arts 53, pp. 461-477.

Hankin, E. H. (1925). The Drawing of Geometric Patterns in Saracenic Art. Memoirs of the Archaeological Society of India, no 15, Government of India. 
Hogendisjk, J.P. (2012). Neugebauer lecture: Mathematicians and Decorative Geometric Tilings in the Medieval Islamic World. Sixth European Congress of Mathematicians, 2 July 2012, Krakow.

Horne, C.E. (2000). Geometric Symmetry in Patterns and Tilings. The Textile Institute, CRC Press.

Kepler, J. (1619). Harmoni Mundi, Lincii.

Koebe, P. (1936). Kontaktprobleme der Konformen Abbildung. Ber. Sächs. Akad. Wiss. Leipzig, Math.-Phys. Kl. 88: 141-164.

Krötenheerdt, O. (1969). Die homogenen Mosaike n-ter Ordnung in der euklidischen Ebene. I, II, III, Wiss. Z. Martin-Luther-Univ. Halle-Wittenberg, Math-Natur. Reihe.

Lu, P. J. and Steinhardt, P. J. (2007a). Decagonal and quasi-crystalline tilings in medieval Islamic architecture, Science 315 (23 Feb 2007), pp. 1106-1110.

Lu, P. J. and Steinhardt, P. J. (2007b). Response to Comment on 'Decagonal and quasicrystalline tilings in medieval Islamic architecture', Science 318 (30 Nov 2007), p. 1383.

Necipoglu, G. (1995). The Topkapı Scroll: Geometry and Ornament in Islamic Architecture.

Getty Center for the History of Art and the Humanities, Getty Center Publication. Santa Monica, $\mathrm{Ca}$.

Özdural, A. (1995). Omar Khayyam, Mathematicians, and Conversazioni with Artisans. Journal of the Society of Architectural Historians, Vol. 54, No. 1, Mar.

Özdural, A. (2000). Mathematics and Arts: Connections between Theory and Practice in the Medieval Islamic World, Historia Mathematica 27, Academic Press, 2000, pp. 171301.

Penrose, R (1974). "The role of aesthetics in pure and applied mathematical research", Bulletin of the Institute of Mathematics and its Applications 10: 266ff.

Prange, S.R. (2009). The Tiles of Infinity. Saudi Aramco World 24-31 of the September/October 2009.

Rull Pérez, F. (1987). La noción de cuasi-cristal a través de los mosaicos árabes', Boletín Sociedad Española de Mineralogía 10 (1987) 291-298. 
Saltzman, P. W. (2008). Quasi-periodicity in Islamic ornamental design, Nexus VII: Architecture and Mathematics, ed. K. Williams, 2008, pp. 153-168.

Sutton, A. (2009). Ruler \& Compass. Ed. Walker Publishing Company. 2009 New York.

Voderberg, H. (1937). Zur Zerlegung der Ebene in kongruente Bereiche in Form einer Spirale. Jahresher. Deutsch. Math.-Verein 47, 159-160.

Received: May 25, 2014 